$50 \%$. Follicular lymphoma has potentially fatal consequences, and the patient died six months post-operatively.

\section{PRIMARY SMALL CELL CARCINOMA OF THE BREAST}

Sara Diab, Ban Sulaiman

Department of Anatomical Pathology, Gosford Hospital, NSW Health Pathology, NSW, Australia

Small cell carcinoma arising in breast is very rare. The diagnosis of primary small cell carcinoma can only be made if a nonmammary site is excluded clinically or if in situ component can be demonstrated histologically as in this case. We reoprt a case of a 68-year-old female patient who presented with a left breast lump, which was diagnosed as a triple negative high grade cancer on core biopsy. The patient received neoadjuvant chemotherapy followed by a left breast lump removal (lumpectomy) with sentinel lymph node biopsy. Macroscopically, there is a well circumscribed tan to pink mass up to $24 \mathrm{~mm}$. Microscopically, the breast shows nests of tumour cells. The tumour cells are small with scant cytoplasm, salt and pepper chromatin, nuclear moulding. Mitotic and apoptotic figures are plentiful. There is ductal carcinoma in situ (DCIS) with similar cytological features. Immunohistochemistry was performed. The tumour cells were positive for E-Cadherin, p53 and CK7, they were weakly positive for NSE and INSM1 and they were negative for CK5/6, S100 and TTF1. Ki67 score was $90 \%$. Features are compatible with primary small cell carcinoma. Small cell carcinoma has a very aggressive behaviour. The management of the primary tumour has not been standardised, due to the rarity of this entity.

\section{SYRINGOCYSTADENOCARCINOMA PAPILLIFERUM: A RARE ADNEXAL SKIN TUMOUR}

Pranav Dorwal ${ }^{1}$, Joel Pinczewski ${ }^{1}$, Jonathan Samuel Levy ${ }^{2}$ ${ }^{1}$ Dorevitch Pathology, Heidelberg, Vic, Australia; and ${ }^{2}$ Skin2, St Leonards, NSW, Australia

Syringocystadenocarcinoma papilliferum (SCACP) is an extremely rare malignant adnexal skin tumour with apocrine differentiation. As per our literature search 25 cases of SCACP have been reported so far. It is the malignant counterpart of the more common adnexal skin tumour, syringocystadenoma papilliferum (SCP). They usually arise in a pre-existing long standing stasyringocystadenoma papilliferum, often in the setting of a naevus sebaceous of Jadassohn. ${ }^{1}$ A 65 -year-old male presented with a history of a scalp lesion that had been growing over a number of years. The H\&E stained sections revealed a reticulated lesion composed of markedly atypical appearing cells with basaloid features. Nests of these atypical cells invaded the superficial dermis. The lesion extended to the base of the specimen. Lymphovascular invasion and perineural spread were not observed. CK7 and p63 immunostains revealed focally strong positive staining within the lesion. These findings support the diagnosis of a syringocystadenocarcinoma papilliferum. We also carried out a brief review of literature and found a total of 26 cases of SCACP. These had mean age of 67 years (range 42-93 years), with slight male predominance with male to female ratio of 1.6:1. The most common site was the scalp (half of the cases).

\section{Reference}

1. Elder DE, Massi D, Scolyer RA, et al., editors. WHO Classification of Skin Tumours. Lyon: International Agency for Research on Cancer, 2018.

\section{COCOON ABDOMEN: BOWELS IN A WEB}

Pranav Dorwal $^{1}$, Robyn Laurie ${ }^{1}$, Kostas Syrrakos ${ }^{2}$

${ }^{1}$ Dorevitch Pathology, Heidelberg, VIC, Australia; and

${ }^{2}$ Mulgrave Private Hospital, VIC, Australia

Cocoon abdomen is a rare, idiopathic condition characterised by fibrous encapsulation of the bowel segments giving an appearance of a cocoon. The patient may present with variable and nonspecific symptoms like abdominal pain, tenderness, vomiting and it sometimes even leads to intestinal obstruction. We hereby report a case of 54-year-old male who presented with symptoms of intestinal obstruction. Apart from the sparing of $60 \mathrm{~mm}$ of the resection margin at either ends, most of the segment was encased by the peritoneum giving a 'spider-web' appearance. On opening the intestinal segment, there were multiple areas of kinking of the wall with narrowing of the lumen in the areas of the 'cocooning' No obvious mass lesion was identified either on the mucosal aspect or in the mesentery. Microscopic examination revealed that multiple loops of small bowel were tethered together by a loose, oedematous fibrovascular membrane. Sclerosing encapsulating peritonitis (SEP) is an extremely rare clinical entity and was first described by Foo et al. in 1978, ${ }^{1}$ in an adolescent girl, which is reported to be the most common age group. Our case was found in a middle-aged male, which differs in both age group and sex of the common incidence of this entity. The aetiology of this condition remains unknown; however there have been cases where the patients have had a prior abdominal surgery, abdominal tuberculosis, liver transplant or recurrent peritonitis. ${ }^{2}$

References

1. Foo KT, Ng KC, Rauff A, et al. Unusual small intestinal obstruction in adolescent girls: The abdominal cocoon. Br J Surg 1978; 65: 427-30.

2. Stanley MM, Reyes CV, Greenlee HB, et al. Peritoneal fibrosis in cirrhotics treated with peritoneovenous shunting for ascites. Digest Dis Sci 1996; 41: 571-7.

\section{A SPINDLE CELL LESION: CASE REPORT OF A RECTAL GIST PRESENTING A PROSTATIC TUMOUR}

Miriam Fewtrell, Mudiwa Muronda

Department of Anatomical Pathology, South Western Area

Pathology Service, Liverpool Hospital, Sydney, NSW, Australia

Gastrointestinal stromal tumour (GIST) most commonly occurs in the stomach, followed by the small bowel and uncommonly the colorectum. GISTs can be composed of spindled or epithelioid cells, with most cases characterised by a gain of function mutation of the KIT gene. This case study reports a patient presenting with urinary retention due to a prostatic mass which was biopsied. The histology was of a mitotically active spindle cell lesion with necrosis. Positive staining for CD117 and DOG1 confirmed the diagnosis of GIST. Subsequent imaging demonstrated a rectal origin of the tumour. 